Research Paper

\title{
Protein chip and bioinformatic analyses of differentially expressed proteins involved in the effect of hydrogen-rich water on myocardial ischemia-reperfusion injury
}

\author{
Liangtong $\mathrm{Li}^{1,4},{ }^{,}$, Tongtong $\mathrm{Liu}^{2},{ }^{*}$, Xiangzi Li ${ }^{1}$, Xuanchen $\mathrm{Liu}^{1}$, Li Liu ${ }^{1}$, Shaochun $\mathrm{Li}^{1}$, Zhilin $\mathrm{Li}^{3}$, Yujuan \\ Zhou $^{1 凶}$, Fulin Liu ${ }^{2}$
}

1. School of Medicine, Hebei University, Baoding, 071000, China

2. Affiliated Hospital of Hebei University, Baoding, 071000, China

3. School of Chemistry, Hebei University, Baoding 071000, China

4. Central Laboratory of Affiliated Hospital of Hebei University, Baoding 071000, China

5. Department of Cardiac Surgery, Affiliated Hospital of Hebei University, Baoding, 071000, China

*These authors contributed equally to this work.

$\triangle$ Corresponding author: Yujuan Zhou, Email: zyj@hbu.edu.cn; Tel.: +86-13513228901 Fulin Liu, Email: lful666@sina.com; Tel.: +86-13930247291

(c) The author(s). This is an open access article distributed under the terms of the Creative Commons Attribution License (https://creativecommons.org/licenses/by/4.0/). See http://ivyspring.com/terms for full terms and conditions.

Received: 2019.04.25; Accepted: 2019.06.30; Published: 2019.08.14

\begin{abstract}
Background: The differentially expressed proteins (DEPs) involved in the effect of hydrogen-rich water on myocardial ischemia reperfusion injury (MIRI) and their biological processes and signaling pathway were analyzed. Methods: 20 Wistar rats were randomly and equally divided into a control and a hydrogen-rich group. Hearts were removed and fixed in a Langendorff device. The control group was perfused with K-R solution, and the hydrogen-rich water group was perfused with K-R solution + hydrogen-rich water. Protein was extracted from the ventricular tissues, and GSR-CAA-67 was used to identify the DEPs between two groups. DEPs were analyzed through bioinformatic methods. Results: Compared with the control group, in the treatment group, the expression of 25 proteins was obviously decreased $(P<0.05)$. For the DEPs, 359 biological processes, including the regulation of signaling pathways, immune reaction and formation of cardiovascular endothelial cells, were selected by $\mathrm{GO}$ enrichment analysis. Five signaling pathways were selected by KEGG pathway enrichment analysis. Conclusions: 25 proteins that are involved in hydrogen-water reducing MIRI were selected by high-throughput GSR-CAA-67. The biological processes and metabolic pathways involved in the DEPs were summarized, providing theoretical evidence for the clinical application of hydrogen-rich water.
\end{abstract}

Key words: hydrogen-rich water; myocardial ischemia-reperfusion injury; protein chip, GO enrichment analysis; KEGG pathway analysis

\section{Introduction}

Heart failure remains one of the most prevalent and challenging medical conditions with high morbidity and mortality despite advances in treatment [1-2]. In recent years, with the advancement of shock treatment and the establishment and application of methods such as thrombolytic therapy, cardiopulmonary bypass, cardiopulmonary cerebral resuscitation, and organ transplantation, the blood circulation of myocardial tissue has been reconstructed, and the function is restored. Due to the repair of damaged tissue structure, the patients' condition can be improved or recovered. However, sometimes reperfusion after ischemia not only fails to restore the function of tissues and organs but also 
aggravates myocardial tissue, cardiac dysfunction and structural damage. This phenomenon of tissue damage and even irreversible damage after ischemic myocardial blood flow recovery is called myocardial ischemia-reperfusion injury (MIRI) [3-4]. MIRI is the most common and serious pathophysiological phenomenon that occurs during the perioperative period that seriously affects the prognosis of patients [5], and methods to effectively treat this disease has attracted increasing attention.

As the study of MIRI increase, an increasing number of drugs are being discovered. In recent years, an element that has been overlooked has gradually become a focus of study: hydrogen; studies have found that hydrogen can attenuate MIRI [6-7]. Liu Xue-cong [6] found that the use of hydrogen-rich water (saturated hydrogen in normal saline) can reduce malonaldehyde (MDA) activity and increase superoxide dismutase (SOD) activity, thereby reducing MIRI and improving cardiac function. At present, the mechanism of action of hydrogen is still in the exploration stage. In this study, a rat model of isolated heart ischemia-reperfusion was established, and a high-throughput G-Series Rat Cytokine Array 67 (GSR-CAA-67) protein chip was used to screen the differentially expressed proteins (DEPs) in the hydrogen-rich water group from the control group. Gene ontology (GO) enrichment and Kyoto Encyclopedia of Genes and Genome (KEGG) pathway enrichment analyses were used to summarize the biological processes and metabolic pathways that the DEPs are involved in, which provides a theoretical basis for the application of hydrogen-rich water in clinical settings.

\section{Materials and Methods}

\subsection{Materials}

\subsubsection{Experimental animals}

Twenty male Wistar rats, weighing 290-320g were provided by Beijing Vital River Laboratory Technology Co., Ltd. (Certificate of Conformity: SCXK (Beijing) 2016-0011). The present study was approved by the Animal Ethical and Welfare Committee of Hebei University (Baoding, China).

\subsubsection{Experimental reagents}

The experimental hydrogen-rich water was provided by Mr. Zhilin Li from the School of Chemistry, Hebei University (the preparation technology has been granted a national patent, patent number ZL102557227B), G-Series Rat Cytokine Array 67kit (GSR-CAA-67, RayBiotech) was used.

\subsubsection{Laboratory apparatus}

InnoScan 300 MicroArray Scanner (Innopsys) was used.

\subsection{Methods}

\subsubsection{Establishment of myocardial ischemia-reperfusion model and groups}

Twenty male Wistar rats were fed a normal chow diet for one week. The rats were randomly divided into a control group and a hydrogen-rich water group, with 10 rats in each group. Sodium pentobarbital $(50 \mathrm{mg} / \mathrm{kg})$ and heparin $(250 \mathrm{U} / \mathrm{kg})$ were injected intraperitoneally according to the body weight of the rats. After anesthesia was effectively administered, the abdominal wall was quickly opened from the lower rib of the xiphoid process, and the diaphragm was revealed. Simultaneously, the anterior line of the iliac crest was cut open and lifted up to the cephalad, without affecting the aorta of the heart. The heart was removed, the aorta was cannulated and fixed in a Langendorff device, and a $37^{\circ} \mathrm{C}$ perfusate with balanced oxygen $\left(95 \% \mathrm{O}_{2}+5 \%\right.$ $\mathrm{CO}_{2}$ ) was pre-perfused at a perfusion pressure of 7.85 $\mathrm{kPa}$. The control group was perfused with K-R solution, and the hydrogen-rich water group was perfused with K-R solution + hydrogen-rich water $(0.6 \mathrm{mmol} / \mathrm{L}, \mathrm{pH} 7.3)$. After reverse perfusion for 10 min, the treatment was administered at room temperature for $20 \mathrm{~min}$, and reperfusion was performed for $20 \mathrm{~min}$. After completion, the rat left ventricular was collected.

\subsubsection{Myocardial samples detected by protein chip}

Rat myocardial tissue was first lysed and then quantified with a BCA assay. Finally, the sample was diluted to $500 \mu \mathrm{g} / \mathrm{ml}$. The slide chip was removed from the box equilibrated at room temperature for 20-30 $\mathrm{min}$, the package was opened, the seal was removed, and the chip was placed in a vacuum desiccator or dried at room temperature for 1-2 $\mathrm{h}$. One hundred microliters of sample dilution was added to each well and incubated for $1 \mathrm{~h}$ on a shaker at room temperature to block the semi-quantitative antibody chip. The buffer $(500 \mu \mathrm{g} / \mathrm{ml}$ loading) in each well was removed, and $100 \mu \mathrm{l}$ of the sample was added to the wells and incubated at $4^{\circ} \mathrm{C}$ overnight. The slides were cleaned by Thermo Scientific Well wash Versa chip washer in two steps. First, 20× Wash I was diluted with deionized water, and the slides were washed 10 times with $250 \mu 1$ of $1 \times$ Wash I per well, oscillating for 10 s with a high oscillation intensity. Then, dilute $20 \times$ wash solution II was diluted with deionized water, and the slides were washed six times with $250 \mu 1$ of $1 \times$ wash solution II per well, oscillating for 10s with a 
high oscillation intensity. The antibody mixture was centrifuged and added to $1.4 \mathrm{ml}$ of the sample dilution, mixed well and centrifuged again quickly. Eighty microliters of the detection antibody was added to each well and incubated for 2 hours on a $37^{\circ} \mathrm{C}$ shaker. Afterwards, the wells were washed. The Cy3-streptavidin tubule was centrifuged, and then 1.4 $\mathrm{ml}$ of the sample dilution was added, mixed well and centrifuge again quickly. Eighty microliters of Cy3-streptavidin was added to each well, and the slides were wrapped in aluminum foil to protect from light and incubated for $1 \mathrm{~h}$ on a $37^{\circ} \mathrm{C}$ shaker. Afterwards, the wells were washed. The signal was scanned by the InnoScan 300 to detect fluorescence using Cy3 channel (excitation frequency $=532 \mathrm{~nm}$ ). Data analysis was performed using GSR-CAA-67 data analysis software.

\subsection{Statistical analysis}

\subsubsection{Raw data normalization}

The raw data obtained by the chip scan was subjected to chip background removal and inter-chip normalization processing by Raybiotech software.

\subsubsection{Screening for differentially expressed proteins}

After the raw data were normalized by the software, the resulting data were selected for analysis. DEPs with $P<0.05$ were first retained and then further screened by Foldchange (expression difference multiple). The selection conditions were as follows: (1)Foldchange $\leq 0.83$ or Foldchange $\geq 1.2$; and (2) Fluorescent signal $>150$.

\subsubsection{Cluster analysis}

For cluster analysis, the heatmap.2 function and gplots package from $R /$ bioconductor were used. The distance between two samples was calculated as the Euclidean distance; the distance between the two clusters was calculated with the furthest neighbor method (complete), and the distance between classes was defined as the maximum distance.

\subsubsection{GO enrichment analysis of DEPs}

Fisher's exact test and the clusterProfiler package from R/bioconductor were used. For selection, the number of genes that differed on a certain GO term/GO was $\geq 2$, and $\mathrm{P}<0.05$.

\subsubsection{KEGG pathway enrichment analysis of DEPs}

Fisher's exact test and the clusterProfiler package from R/bioconductor were used. For selection, the number of genes that differed on a certain term/ pathway was $\geq 5$, and $\mathrm{P}<0.05$.

\section{Results}

\subsection{Establishment of DEPs profiles}

Through the screening of DEPs in the control group and the hydrogen-rich water group, we obtained 25 DEPs (Table 1), and all DEPs in the hydrogen-rich water group (group C) were down-regulated compared with those in the control group (group $\mathrm{H}$ ). By querying the information on these 25 proteins, most of them were found to be related to the inflammatory response [6].

Table 1. The differentially expressed proteins between the case group and control group

\begin{tabular}{|c|c|c|c|}
\hline Protein name & Gene ID & $\begin{array}{l}\text { Foldchange } \\
\text { (the group } \mathrm{C} / \text { the group } \mathrm{H} \text { ) }\end{array}$ & $P$ \\
\hline Gas 1 & 683470 & 0.67366 & 0.000045 \\
\hline Flt-3L & 103691134 & 0.66336 & 0.000088 \\
\hline IL-17F & 301291 & 0.65264 & 0.000154 \\
\hline Galectin-1 & 56646 & 0.72155 & 0.000775 \\
\hline JAM-A & 116479 & 0.70737 & 0.000943 \\
\hline TIM-1 & 286934 & 0.64563 & 0.028159 \\
\hline Adiponectin & 246253 & 0.65508 & 0.002857 \\
\hline IL-3 & 24495 & 0.81244 & 0.003768 \\
\hline Erythropoietin & 24335 & 0.75290 & 0.003970 \\
\hline $4-1 \mathrm{BB}$ & 500590 & 0.67767 & 0.005473 \\
\hline MIP-1a & 25542 & 0.82481 & 0.006305 \\
\hline B7-1 & 25408 & 0.81611 & 0.006446 \\
\hline GFR alpha-1 & 25454 & 0.80775 & 0.009870 \\
\hline IL-2 Ra & 25704 & 0.68152 & 0.011098 \\
\hline Notch-1 & 25496 & 0.77854 & 0.013427 \\
\hline Fractalkine & 89808 & 0.68499 & 0.015341 \\
\hline IL-22 & 500836 & 0.59740 & 0.015728 \\
\hline P-Cadherin & 116777 & 0.70260 & 0.017220 \\
\hline Decorin & 29139 & 0.72011 & 0.020027 \\
\hline Nope & 363081 & 0.76400 & 0.020463 \\
\hline CINC-3 & 114105 & 0.75123 & 0.024528 \\
\hline CD48 & 245962 & 0.82264 & 0.027651 \\
\hline TIMP-1 & 116510 & 0.64563 & 0.002634 \\
\hline Prolactin & 24683 & 0.75411 & 0.036032 \\
\hline Prolactin R & 24684 & 0.75411 & 0.045943 \\
\hline
\end{tabular}

\subsection{Clustering Analysis of DEPs}

By plotting the cluster heatmap, the global expression of these 20 samples and 25 DEPs can be presented from a macroscopic perspective. Figure 1 visually shows that the expression of DEPs in the control group (group $\mathrm{H}$ ) and the hydrogen-rich water group (group C) are significantly different, and the DEPs in the hydrogen-rich water group are down-regulated relative to that in the control group.

\subsection{GO Enrichment Analysis of DEPs}

A total of 359 biological processes were screened in this experiment, and these processed involved signaling pathway regulation, immune response, and cardiovascular endothelial cell formation. Table 2 shows some of the results from this experiment (the biological processes in Table 2 are ranked in descending order of size according to the number of 
enriched genes (Count), taking the first 10 results). From Table 2, we found that six of these ten biological processes are related to the JAK-STAT pathway, namely, the STAT cascade, JAK-STAT cascade, regulation of the STAT cascade, regulation of JAK-STAT cascade, positive regulation of STAT cascade, and positive regulation of the JAK-STAT cascade.

\subsection{KEGG Pathway Enrichment Analysis of DEPs}

A total of 5 pathways were screened in this experiment. Table 3 shows the results from this experiment (the obtained pathways in Table 3 are sorted in descending order of magnitude according to the Count value).

\section{Discussion}

MIRI has a relatively complex mechanism, and its pathological process involves physiological mechanisms such as the inflammatory response, oxidative stress, and intracellular calcium overload [8-11]. As a kind of element widely distributed in nature, hydrogen has a wide range of physiological effects such as anti-oxidation effects and the selective scavenging of free radicals [12]. Presently, many scholars are studying the mechanism of hydrogen alleviation in ischemia-reperfusion injury tissues. Recent research found that hydrogen-rich water can reduce the expression of TNF-a during ischemia-reperfusion [13] and reduce myocardial infarct size [14]. Gao $Y$ et al [15] found that hydrogen-rich water can reduce endoplasmic reticulum stress, apoptosis and MIRI in rats. Presently, the protection mechanism of MIRI for hydrogen-rich water is still unclear.

Table 2. The GO terms that are enriched with the differentially expressed proteins

\begin{tabular}{llll}
\hline GO terms & Gene Ratio $^{*}$ & Count & $p$-adjust \\
\hline cytokine-mediated signaling pathway & $9 / 24$ & 9 & $5.1772 \times 10^{-7}$ \\
response to molecule of bacterial origin & $8 / 24$ & 8 & 0.0000103 \\
response to lipopolysaccharide & $8 / 24$ & 8 & 0.0000099 \\
STAT cascade & $7 / 24$ & 7 & $5.7571 \times 10^{-7}$ \\
JAK-STAT cascade & $7 / 24$ & 7 & $5.75708 \times 10^{-7}$ \\
regulation of STAT cascade & $6 / 24$ & 6 & 0.0000099 \\
regulation of JAK-STAT cascade & $6 / 24$ & 6 & 0.0000099 \\
positive regulation of leukocyte proliferation & $5 / 24$ & 5 & 0.0002004 \\
positive regulation of STAT cascade & $5 / 24$ & 5 & 0.0000099 \\
positive regulation of JAK-STAT cascade & $5 / 24$ & 5 & 0.0000099 \\
\hline
\end{tabular}

* GeneRatio is the number of differential genes corresponding to GO term/the number of differential genes that can correspond to the same type in the GO database.

\# Count is the number of enriched genes.
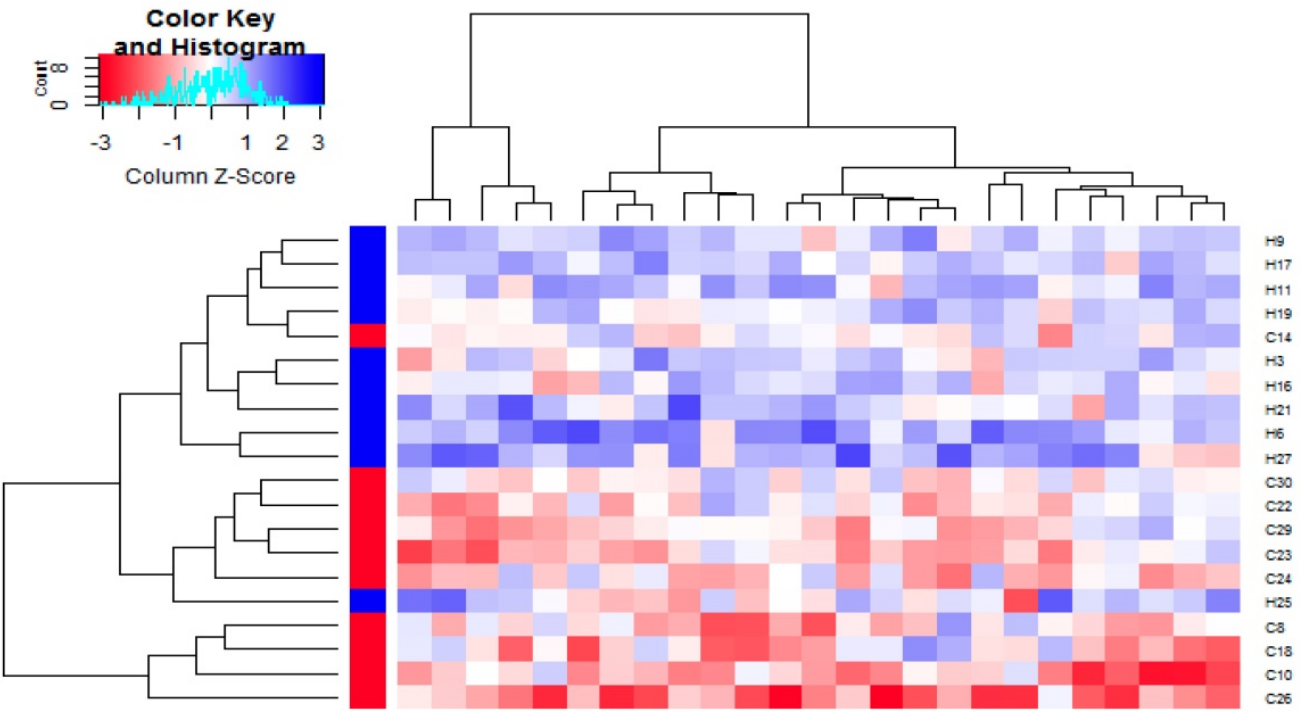

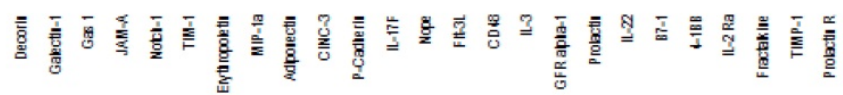

Figure 1. Clustering analysis of the differentially expressed proteins between group $\mathrm{C}$ and group $\mathrm{H}$

Table 3. The KEGG pathway terms those are enriched with the differentially expressed proteins

\begin{tabular}{|c|c|c|c|}
\hline KEGG pathway & Gene ID & Count ${ }^{*}$ & P.adjust \\
\hline Cytokine-cytokine receptor interaction & $114105 / 89808 / 24684 / 500590 / 24335 / 103691134 / 25704 / 24495 / 301291 / 500836 / 25542 / 24683$ & 12 & $2.00915 \times 10^{-12}$ \\
\hline JAK-STAT signaling pathway & $24684 / 24335 / 25704 / 24495 / 500836 / 24683$ & 6 & 0.000037 \\
\hline Hematopoietic cell lineage & $24335 / 103691134 / 25704 / 24495$ & 4 & 0.001694 \\
\hline PI3K-Akt signaling pathway & $24684 / 24335 / 25704 / 24495 / 24683$ & 5 & 0.017927 \\
\hline Th17 cell differentiation & 25704/301291/500836 & 3 & 0.027910 \\
\hline
\end{tabular}

"Count is the number of enriched genes. 
High-throughput protein chips play an important role in scientific research due to its richer target factor detection and smaller sample volume requirement. It is widely used in the research of disease mechanisms [16-18]. Recently, Bharath C [19] used the Raybiotech human AAH-APO-1 to study the molecular mechanism of cerebral ischemic apoptosis, and comprehensively revealed the changes of pro-apoptotic factors and anti-apoptotic expressed protein profiles in cerebral ischemia-reperfusion. This finding provides an important reference for further research on ischemic diseases. In this experiment, we used the GSR-CAA- 67 protein chip from Raybiotech, USA, that can detect 67 proteins in rats. This protein chip was used to detect DEPs between the hydrogen-rich water group and the control group by constructing a rat isolated heart langendorff model. A total of 25 DEPs were screened out $(P<0.05)$. Compared with their levels in the control group, the 25 DEPs in the hydrogen-rich water group were all down-regulated. This provides a basis for exploring the mechanism of action of hydrogen-rich water and finding a target for hydrogen-rich water.

Studies have shown that reducing inflammation can attenuate MIRI [20]. Most of the 25 down-regulated proteins screened in this experiment were related to the inflammatory response, and it can be preliminarily concluded that hydrogen-rich water may protect the myocardium from damage by reducing the inflammatory response. The results of KEGG pathway enrichment analysis demonstrate that Th17 differentiation is related to the protective effect of hydrogen-rich water. Th17 cells are a subset of T cells associated with many inflammatory and autoimmune diseases that secrete inflammatory factors such as IL-17 and IL-22 [21-22]. There is data showing that Th17 cells are closely related to the occurrence of renal ischemia-reperfusion injury, and its expression is significantly increased in ischemia-reperfusion tissues [23]. Table 1 and 3 show that the proteins involved in the differentiation of Th17 cells include IL-2Ra, IL-17F and IL-22, and the expression levels of these three proteins are decreased compared with those of the control group. Therefore, we speculate that the reason for the reduction of MIRI by hydrogen-rich water may be related to the inhibition of Th17 cell differentiation; however, the results need further verification.

By comparing the results of GO enrichment analysis and KEGG pathway enrichment analysis, we found that the JAK-STAT signaling pathway was enriched by both methods, indicating that the JAK-STAT pathway may be involved in the mechanism of action of hydrogen-rich water. The JAK-STAT signaling pathway has been studied in recent years, and it is widely involved in biological processes such as cell proliferation [24], differentiation [25], and the inflammatory response [26]. The basic process of this pathway is as follows: cytokines bind to their corresponding receptors causing receptor dimerization $\rightarrow$ activation of JAKs $\rightarrow$ phosphorylation of STATs $\rightarrow$ STATs form dimers and enter the nucleus $\rightarrow$ STATs form dimers binding to the target gene promoter and then regulate gene expression [27]. Some experimental studies have shown that the JAK-STAT pathway is closely related to myocardial ischemia-reperfusion, but its specific role is still unknown. Oh YB et al [28] constructed a rat isolate heart perfusion model and used a JAK3-specific blocker, JANEX-1, to block the JAK-STAT pathway and explore whether it would aggravate ischemia-reperfusion injury. They found that myocardial damage was significantly attenuated after blocking the JAK-STAT pathway, suggesting that activation of JAK-STAT is unfavorable for MIRI. However, a recent study had different results. Mudaliar $\mathrm{H}$ et al [29] found that remote ischemic preconditioning can attenuate early growth response-1 (EGR-1) expression, which is a key upstream activator in a variety of cardiovascular diseases, through the activation of the JAK-STAT pathway, thereby reducing MIRI. We have conducted in-depth research on the JAK-STAT [8] and PI3K-AKT pathway, and found that hydrogen-rich water can up-regulate the JAK-STAT and PI3K-AKT signaling pathway and alleviate MIRI in rats. The results showed that the P-JAK2/JAK2, P-STAT3/STAT3 and p-AKT/AKT increased when compared to the control, while the P-STAT1/STAT1 decreased in the hydrogen-rich water group. In addition, the apoptosis rate of the hydrogen-rich water group decreased significantly.

The GSR-CAA-67 protein chip used in this experiment can detect the expression level of only proteins, but there is no test for the modification of proteins. This requires in-depth research to be conducted in order to determine the target of hydrogen-rich water for MIRI.

\section{Abbreviations}

DEPs: differentially expressed proteins; MIRI: myocardial ischemia reperfusion injury; MDA: malonaldehyde; SOD: superoxide dismutase; GSR-CAA-67: G-Series Rat Cytokine Array 67; GO: Gene ontology; KEGG: Kyoto Encyclopedia of Genes and Genome.

\section{Acknowledgements}

This article was supported financially by the Medical Science Research Key Project Foundation of 
Hebei Province (No. 20130369).

\section{Author Contributions}

Conceptualization, Y.Z., F.L., L.L. and T.L.; formal analysis, L.L., T.L., X.L. and X.L., methodology, Y.Z., F.L., L.L., T.L., X.L., X.L. and Z.L.; project administration, L.L., T.L., X.L., X.L. and Z.L.; validation, L.L. and S.L., writing, original draft, L.L., T.L., X.L. and X.L.; writing, review and editing L.L., S.L., Y.Z. and F.L.

\section{Competing Interests}

The authors have declared that no competing interest exists.

\section{References}

1. Pohl J, Hendgen-Cotta UB, Stock $P$, et al. Myocardial Expression of Macrophage Migration Inhibitory Factor in Patients with Heart Failure. J Clin Med. 2017; 6: 95.

2. Stroethoff M, Behmenburg F, Meierkord S, et al. Cardioprotective Properties of Omecamtiv Mecarbil against Ischemia and Reperfusion Injury. J Clin Med. 2019; 8: 375

3. Aghaei M, Motallebnezhad M, Ghorghanlu S, et al. Targeting autophagy in cardiac ischemia/reperfusion injury: A novel therapeutic strategy. J Cell Physiol. 2019; 234: 16768-78.

4. Shin B, Cowan DB, Emani SM, et al. Mitochondrial Transplantation in Myocardial Ischemia and Reperfusion Injury. Adv Exp Med Biol. 2017; 982: 595-619.

5. Horton JL, Virag J. Use of Multifactorial Treatments to Address the Challenge of Translating Experimental Myocardial Infarct Reduction Strategies. Int J Mol Sci. 2019; 20: 1449 .

6. Liu X, Liu F, Li Z, et al. Protective effects of hydrogen saturated saline alleviating myocardial ischemia reperfusion injury in rats. Shandong Medical Journal. 2015; 55: 1-3

7. Hayashida K, Sano M, Ohsawa I, et al. Inhalation of hydrogen gas reduces infarct size in the rat model of myocardial ischemia-reperfusion injury. Biochemical and Biophysical Research Communications. 2008; 373: 30-5

8. $\mathrm{Li} \mathrm{X}, \mathrm{Li} \mathrm{L}$, Liu X, et al. Attenuation of cardiac ischaemia-reperfusion injury by treatment with hydrogen-rich water. Curr Mol Med. 2019; 294-302.

9. Zhang HM, Tang DL, Tong L, et al. Gualou xiebai banxia decoction inhibits NF-kappa B-dependent inflammation in myocardial ischemia-reperfusion injury in Rats. J Tradit Chin Med. 2011; 31: 338-43.

10. Yang $\mathrm{M}$, Chen J, Zhao J, et al. Etanercept attenuates myocardial ischemia/reperfusion injury by decreasing inflammation and oxidative stress. PLoS One. 2014; 9: e108024.

11. Pittas K, Vrachatis DA, Angelidis C, et al. The Role of Calcium Handling Mechanisms in Reperfusion Injury. Curr Pharm Des. 2018; 24: 4077-89.

12. Wang Z, Liu X, Liu F, et al. The protective of hydrogen-rich water on myocardial ischemia reperfusion injury. Journal of Clinical Cardiology. 2017; 33: 596-600

13. Lin $\mathrm{CP}$, Chuang WC, Lu FJ, et al. Anti-oxidant and anti-inflammatory effects of hydrogen-rich water alleviate ethanol-induced fatty liver in mice. World J Gastroenterol. 2017; 23: 4920-34

14. Feng R, Cai M, Wang X, et al. Early Aerobic Exercise Combined with Hydrogen-Rich Saline as Preconditioning Protects Myocardial Injury Induced by Acute Myocardial Infarction in Rats. Appl Biochem Biotechnol. 2019; 187: 663-76.

15. Gao Y, Yang H, Chi J, et al. Hydrogen Gas Attenuates Myocardial Ischemia Reperfusion Injury Independent of Postconditioning in Rats by Attenuating Endoplasmic Reticulum Stress-Induced Autophagy. Cell Physiol Biochem. 2017; 43: 1503-14.

16. Yuan $\mathrm{Y}$, Lin ZT, Wang H, et al. Protein Arrays I: Antibody Arrays. Methods Mol Biol. 2017; 1654: 261-9.

17. Yuan Y, Wang H, Lin ZT, et al. Protein Arrays II: Antigen Arrays. Methods Mol Biol. 2017; 1654: 271-7.

18. Yuan Y, Hong X, Lin ZT, et al. Protein Arrays III: Reverse-Phase Protein Arrays. Methods Mol Biol. 2017; 1654: 279-89.

19. Chelluboina B, Klopfenstein JD, Gujrati $M$, et al. Temporal regulation of apoptotic and anti-apoptotic molecules after middle cerebral rrtery occlusion followed by reperfusion, Mol Neurobiol. 2014; 49: 50-65.

20. Sheibani M, Faghir-Ghanesefat H, Dehpour S, et al. Sumatriptan protects against myocardial ischaemia-reperfusion injury by inhibition of inflammation in rat model. Inflammopharmacology. 2019; doi: 10.1007/s10787-019-00586-5.
21. Affandi AJ, Silva-Cardoso SC, Garcia S, et al CXCL4 is a novel inducer of human Th17 cells and correlates with IL-17 and IL-22 in psoriatic arthritis. Eur J Immunol. 2018; 48: 522-31.

22. Martinez-Lopez M, Iborra S, Conde-Garrosa R, et al. Microbiota Sensing by Mincle-Syk Axis in Dendritic Cells Regulates Interleukin-17 and -22 Production and Promotes Intestinal Barrier Integrity. Immunity. 2019; 50: 446-61, e9.

23. Lee JW, Bae E, Kwon SH, et al. Transcriptional modulation of the T helper $17 /$ interleukin 17 axis ameliorates renal ischemia-reperfusion injury. Nephrol Dial Transplant. 2018; doi: 10.1093/ndt/gfy370.

24. Groner B, von Manstein V. Jak Stat signaling and cancer: Opportunities, benefits and side effects of targeted inhibition. Mol Cell Endocrinol. 2017; 451: 1-14.

25. Liu K, Tian T, Zheng Y, et al. Scutellarin inhibits proliferation and invasion of hepatocellular carcinoma cells via down-regulation of JAK2/STAT3 pathway. J Cell Mol Med. 2019; 23: 3040-4.

26. Ning SL, Zhu H, Shao J, et al. MiR-21 inhibitor improves locomotor function recovery by inhibiting IL-6R/JAK-STAT pathway-mediated inflammation after spinal cord injury in model of rat. Eur Rev Med Pharmacol Sci. 2019; 23: 433-40.

27. Heim MH. The Jak-STAT pathway: cytokine signalling from the receptor to the nucleus. J Recept Signal Transduct Res. 1999; 19: 75-120.

28. Oh YB, Ahn M, Lee SM, et al. Inhibition of Janus activated kinase-3 protects against myocardial ischemia and reperfusion injury in mice. Exp Mol Med. 2013; 45: e23.

29. Mudaliar H, Rayner B, Billah M, et al. Remote ischemic preconditioning attenuates EGR-1 expression following myocardial ischemia reperfusion injury through activation of the JAK-STAT pathway. Int J Cardiol. 2017; 228: 729-41. 